\title{
Synthetic Images for Evaluating Topographic Correction Algorithm
}

\author{
Ion Sola, María González-Audícana, Jesus Álvarez-Mozos, and José Luis Torres
}

\begin{abstract}
In the last years, many topographic correction (TOC) methods have been proposed to correct the illumination differences between the areas observed by optical remote sensors. Although the available number of TOC methods is high, the evaluation of their performance generally relies on the existence of precise land-cover information, and a standardized and objective evaluation procedure has not been proposed yet. In this paper, we propose an objective procedure to assess the accuracy of these TOC methods on the basis of simulated scenes, i.e., synthetically generated images. These images represent the radiance an optical sensor would receive under specific geometric and temporal acquisition conditions and assuming a certain land-cover type. A simplified method for creating synthetic images using the stateof-the-art irradiance models is proposed, both considering the real topography of a certain area [synthetic real (SR) image] or considering the relief of this area as being completely flat [synthetic horizontal image ( $\mathrm{SH})$ ]. The comparison between the corrected image obtained by applying a TOC method to the SR and $\mathrm{SH}$ images of the same area, allows assessing the performance of each TOC algorithm. This comparison is quantitatively carried out using the structural similarity index. The proposed TOC evaluation procedure is applied to a specific case study in northern Spain to explain its implementation and demonstrate its potential. The procedure proposed in this paper could be also used to assess the behavior of TOC methods operating under different scenarios considering diverse topographic, geometrical, and temporal acquisition configurations.
\end{abstract}

Index Terms-Irradiance, synthetic image, topographic correction (TOC).

\section{INTRODUCTION}

$\mathbf{T}$ HE irradiance impinging on a certain point at the Earth's surface depends on the solar zenith and azimuth angles as well as on the slope and aspect of the terrain, which determine the solar incidence angle $\left(\gamma_{i}\right)$ between the sun rays and the normal to the ground. Differences in the solar incidence angle, i.e., differences in the solar illumination, normally result in variations in the radiance detected by remote sensors between areas with similar land cover and biophysicalstructural properties [1]. This effect can adversely affect the usefulness of remote sensing data for different applications, such as land-use/land-cover mapping, vegetation cover monitoring, change detection, or biophysical parameter estimation, especially in mountainous areas [2]-[6]. The objective of

Manuscript received March 5, 2012; revised November 27, 2012 and February 12, 2013; accepted March 12, 2013. This work was supported in part by the Department of Industry of the Government of Navarre (Spain).

The authors are with the Department of Projects and Rural Engineering, Public University of Navarre, Pamplona 31006, Spain (e-mail: ion.sola@unavarra.es; maria.audicana@unavarra.es; jesus.alvarez@ unavarra.es; jlte@unavarra.es).

Digital Object Identifier 10.1109/TGRS.2013.2255296 topographic correction (TOC) methods is to compensate the differences in radiance between sunny and shaded areas caused by variations in the shape and aspect of terrain. In this paper, a new procedure to assess the performance of TOC algorithms using synthetic images is proposed. This paper is structured as follows. Section II reviews the basis of TOC methods and the evaluation procedures used normally. Section III describes the model used to create synthetic images and the quality index used to assess the TOC correction. Next, a case study is presented in Section IV, where the technique proposed is applied and evaluated. Section V evaluates the performance of four selected TOC algorithms based on the procedure proposed and shows the results obtained. Finally, the main conclusions are drawn in Section VI.

\section{PREVIOUS WORKS}

\section{A. TOC Algorithms}

The topographic effect has a significant impact on the quantitative analysis of remotely sensed data [7]. During the last two decades, several procedures were proposed to correct or attenuate it. Most of these procedures require the computation of the illumination conditions of the area to be corrected [1], [2], [8]-[11]. In those methods, the illumination conditions for each pixel are normally estimated using the cosine of the solar incidence angle, $\cos \gamma_{i}$, which can be calculated from the solar zenith and azimuth angles and the slope and aspect, computed for each pixel using a digital elevation model (DEM)

$$
\cos \gamma_{i}=\cos \beta \cos \theta_{s}+\sin \beta \sin \theta_{s} \cos \left(\varphi_{n}-\varphi_{s}\right)
$$

where $\beta$ is the slope angle, $\varphi_{n}$ is the aspect angle, $\theta_{s}$ is the solar zenith angle, and $\varphi_{s}$ is the solar azimuth angle. Both $\beta$ and $\varphi_{n}$ are computed from the DEM.

TOC methods can be grouped into two subcategories, Lambertian methods (LTOC) and nonLambertian methods (NLTOC), depending on whether they assume reflectance as being independent or not of observation and incidence angles. The simplest and one of the most widely used LTOC is the cosine method, originally proposed by Smith et al. [3] and later modified by Teillet [4]. Alternatively, Civco [5] proposed an improved version considering average illumination conditions.

The cosine method assumes the incident radiation as being reflected in all directions equally. In addition, this method only models the direct portion of the irradiance, even if areas under low-illumination conditions get a considerable proportion of diffuse irradiance. On these areas, the cosine correction has shown a problem of overcorrection [6], [8], [10]-[13]. 
To account for the shortcomings of these unrealistic assumptions, several semiempiric nonLambertian methods are developed including band-dependent parameters, i.e., the Minnaert correction method [3], [4], [14] and the $C$-correction method [4]. The former includes a constant modeling of the nonLambertian behavior of each land cover for every band. The latter introduces, in order to emulate the effect of diffuse irradiance from the sky, a parameter $C$, which is the ratio between the slope and intercept of the linear regression equation between the radiance of each band and $\cos \gamma_{i}$. Similarly, and following this paper of Teillet [4], Soenen et al. [1] proposed the SCS+C correction, where the LTOC method proposed by $\mathrm{Gu}$ and Gillespie [15] for forested areas, so called SCS, was modified to account for diffuse atmospheric irradiance by introducing the previously mentioned parameter $C$.

All the $\mathrm{SCS}+\mathrm{C}$, Minnaert, and $C$-correction methods are physically based, and consist of photometric functions modified using parameters estimated empirically. Nevertheless, purely empiric approaches are also proposed, i.e., the empirical-statistical method of Teillet [4], which assumes a linear relationship between the radiance of each band and $\cos \gamma_{i}$, or the variable empirical coefficient algorithm, proposed by Zhang and Gao [16], including an empirically estimated adjustment factor. Alternatively, many authors have proposed modifications in TOC methods to improve their performance, based on slope-smoothing [12], [17], or on the use of different correction approaches for infrared and visible bands [18], [19].

The results obtained through the NLTOC methods described here are reported to improve if stratifications were applied previous to the TOC correction to more precisely estimate the correction factors. The stratification may be based on the different nonLambertian behavior, i.e., the different surfaceroughness of the land covers on the image to be corrected [10], [20]-[23], the illumination conditions [24]-[26], the terrain slope [7], [11], the terrain orientation [5], [26], or a combination of any of these factors [19], [25], [27].

\section{B. Assessment of the Quality of TOC Correction Methods}

An essential point, necessary to evaluate objectively and accurately the different TOC methods, is the analysis of the quality of the corrected images. With this aim, traditionally an evaluation based on the visual assessment of the removal of the topographic effect in satellite imagery is proposed [5], [15], [28], [29]. This approach gives a good first indication on the quality of the correction. However, it is indeed subjective and the assessment strongly depends on the skill of the observer.

A more objective assessment, and one of the most widely used evaluation methods, is the quantification of the reduction of the dependence between $\cos \gamma_{i}$ and the radiance of each spectral band after the correction, measured through both the correlation coefficient or the slope of their linear regression, being $\cos \gamma_{i}$ the independent variable [30]. Such dependence tends to disappear in the TOC-corrected images, being in these cases, both the correlation coefficient and the slope of the regression close to zero, showing that illumination dependence on reflectance values is successfully removed.
This evaluation implicitly assumes land-cover distribution (and hence reflectance) as being independent on terrain slope and aspect. Obviously, this assumption is not valid in areas where slope orientation determines the land-cover. Therefore, in such areas a residual correlation between reflectance and $\cos \gamma_{i}$ is expected, even after a successful TOC [10].

Civco [5] proposed, as an evaluation approach, the analysis of the variations in the radiometry of the corrected scenes. Ideally the overall mean response of the original image should not change after TOC; otherwise the TOC method would have caused an under or overcorrection. Similarly, other authors [7], [12], [31] proposed that the quality of TOCs could be best evaluated by measuring the reduction of the land-cover class variability, measured through the standard deviation of the reflectance within each surface cover class. A perfect correction would result in more homogenous classes with a reduced variability. This assessment method is probably the most objective and quantitatively measurable criterion. However, the reduction of land-cover class variability in topographically corrected imagery is restricted to cases where a priori knowledge of land-cover distributions is available.

Many authors considered the improvement on classification accuracy after TOC as an adequate procedure to assess the goodness of the TOC correction [4], [29]. A classification based on TOC-corrected images should ideally yield a higher accuracy than one using uncorrected data. A similar approach is to evaluate the improvement in biophysical parameter retrievals [21], [24]. However, classification and biophysical parameter estimation assessments carry their own uncertainties in both classification and retrieval algorithms and are unable to directly quantify the degree to which the topographic effect has been reduced.

Alternatively, Hantson and Chuvieco [10] proposed to quantify the increase in temporal stability of a time series for individual pixels, which would represent the robustness of the TOC algorithms under different conditions over time. This option may not be adequate in all cases, being difficult to discern between the temporal variations of spectral response of land-covers and an ineffective correction of the topographic effect, with the risk of excessively homogenizing the image.

\section{Synthetic IMAGES}

We propose the use of synthetic imagery to quantitatively evaluate TOC algorithms. Synthetic images represent the radiance an optical sensor would receive under specific geometric and temporal acquisition conditions, considering a certain land-cover structure and assuming several simplifications.

Synthetic images, on the basis of the Lambertian reflectance law, can be generated considering the real topography of a specific area (synthetic real (SR) image), or considering a perfectly flat surface (synthetic horizontal (SH) image). The latter is the image that should ideally be obtained after successfully removing the topographic effect from the SR image. The comparison between the corrected image obtained applying a TOC method to a SR image and the SH image of the same area, provides a means of objectively assessing the accuracy of the TOC method applied. 
TABLE I

SUMMARY OF SCIENTIFIC AND TECHNICAL NOTATION

\begin{tabular}{|c|c|c|}
\hline Symbol & Parameter & Units \\
\hline$\gamma_{i}$ & solar incidence angle & degree \\
\hline$\beta$ & pixel's slope angle & degree \\
\hline$\theta_{s}$ & solar zenith angle & degree \\
\hline$\varphi_{n}$ & pixel's aspect angle & degree \\
\hline$\varphi_{s}$ & solar azimuth angle & degree \\
\hline$E_{E S N O}$ & extraterrestrial normal irradiance & $\mathrm{W} / \mathrm{m}^{2}$ \\
\hline$m$ & relative optical air mass & -- \\
\hline$a_{e r}$ & optical thickness of a Rayleigh atmosphere & -- \\
\hline$T_{e L(2)}$ & Linke's turbidity factor & -- \\
\hline$\rho$ & land-cover reflectance & -- \\
\hline$T_{u}$ & upward atmospheric transmittance & -- \\
\hline$d$ & Sun-to-Earth distance correction factor & -- \\
\hline$\theta_{0}$ & viewing angle of the satellite & degree \\
\hline$x_{1}, x_{2}, x_{3}$ & $\%$ of irradiance corresponding to $0.5-0.9 \mu \mathrm{m}$ & --- \\
\hline$E_{e, s}$ & direct horizontal irradiance & $\mathrm{W} / \mathrm{m}^{2}$ \\
\hline$E_{e, d}$ & diffuse horizontal irradiance & $\mathrm{W} / \mathrm{m}^{2}$ \\
\hline$E_{e, g}$ & global horizontal irradiance & $\mathrm{W} / \mathrm{m}^{2}$ \\
\hline$E_{\beta, s}$ & direct tilted irradiance & $\mathrm{W} / \mathrm{m}^{2}$ \\
\hline$E_{\beta, r}$ & ground-reflected irradiance & $\mathrm{W} / \mathrm{m}^{2}$ \\
\hline$E_{\beta, d}$ & sky diffuse irradiance & $\mathrm{W} / \mathrm{m}^{2}$ \\
\hline$E_{\beta, g}$ & global tilted irradiance & $\mathrm{W} / \mathrm{m}^{2}$ \\
\hline$L_{s e n}$ & at-sensor radiance & $\mathrm{W} / \mathrm{m}^{2}$.sr. \\
\hline $\mathrm{SH}$ & synthetic image considering flat topography & $\mathrm{W} / \mathrm{m}^{2} . \mathrm{sr}$. \\
\hline$S R$ & synthetic image considering its real relief & $\mathrm{W} / \mathrm{m}^{2}$.sr. \\
\hline$\mu_{x}$ & mean radiance of reference image & $\mathrm{W} / \mathrm{m}^{2} \cdot \mathrm{sr}$. \\
\hline$\mu_{y}$ & mean radiance of corrected image & $\mathrm{W} / \mathrm{m}^{2} \cdot \mathrm{sr}$. \\
\hline$\sigma_{x}$ & standard deviation of reference image & $\mathrm{W} / \mathrm{m}^{2}$.sr. \\
\hline$\sigma_{y}$ & standard deviation of corrected image & $\mathrm{W} / \mathrm{m}^{2} . \mathrm{sr}$. \\
\hline$V_{t}$ & Terrain View Factor & -- \\
\hline$V_{d}$ & Sky View Factor & -- \\
\hline$A I$ & anisotropy index & -- \\
\hline$\Theta$ & binary factor to model cast shadows & -- \\
\hline$\rho_{a d j}$ & average reflectance & -- \\
\hline$E_{e, g a d j}$ & global average horizontal irradiance & $\mathrm{W} / \mathrm{m}^{2}$ \\
\hline$L_{e}$ & direct radiance & $\mathrm{W} / \mathrm{m}^{2}$.sr. \\
\hline$L_{p}$ & path radiance & $\mathrm{W} / \mathrm{m}^{2}$.sr. \\
\hline$\rho_{a}^{\prime}$ & atmospheric albedo & -- \\
\hline
\end{tabular}

The approach proposed here allows simulating synthetic images considering different topographic, geometric, and temporal configurations, as well as different land-cover distributions. Therefore, the influence of acquisition conditions on the behavior of TOC methods can also be explored.

In short, the evaluation approach proposed here is based on the synthetic generation of the image a sensor would acquire for any given area, considering its topography completely flat. This image can be then used as a reference to compare against images corrected with different TOC, using quantitative indexes, in a rigorous, objective, and consistent manner. In the next subsections, the process proposed to generate synthetic images is explained in detail.

\section{A. Synthetic Image Generation}

During the last years, several complete and realistic physicsbased scene simulators are proposed for a great variety of tasks, i.e., the design of systems, the development of data processing algorithms or the understanding of the image formation process [32]. Scenes simulators such as SENSOR, proposed by Börner et al. [33], DIRSIG [34], or the approach proposed by Guanter et al. [35] allows computationally demanding but very realistic modeling of the at-sensor radiance. However, for our particular application a simplified simulation model, which adequately represents the influence of topography on the image acquisition process, is presented. Several simplifications can be adopted to facilitate the process of generating synthetic images. In this paper, we assume a panchromatic sensor working in the $0.500-0.900 \mu \mathrm{m}$ spectrum range, with a constant spectral response function for the whole wavelength range. The main parameters of the synthetic image generation model are summarized in Table I.

The process to simulate a synthetic image for a specific area (Fig. 1) can be summarized in two phases. First, the image representing the global irradiance on each point of the area of interest at a certain date and time is obtained. In the second phase, the top-of-atmosphere radiance based on a surface reflectance map and a certain sensor configuration is generated. This is the final synthetic image.

To obtain the global irradiance at each point of the Earth surface it is necessary to initially estimate the global horizontal irradiance $\left(E_{e, g}\right)$, i.e., the total amount of direct and diffuse radiation reaching the Earth surface, considering it horizontal, in cloudless conditions. Several models can be used to estimate $E_{e, g}$ and its diffuse and direct components [36]-[39]. In this paper, the cloud-free global radiation model [40] is used. This model is validated using 25 test sites spread across Europe within the SATEL-LIGHT project [41]. It shows a good correspondence between estimated and measured values under sunny or quasi-sunny conditions.

$E_{e, g}$ is computed for a specific area, date, and time as the sum of its direct and diffuse components. The direct component $\left(E_{e, s}\right)$ is calculated using the equation of Page [40] and the diffuse component $\left(E_{e, d}\right)$ is calculated using the equation of Dumortier [42]

$$
\begin{aligned}
E_{e, g}= & E_{e, d}+E_{e, s} \\
E_{e, s}= & x_{1} E_{\mathrm{ESNO}} \cos \theta_{s} \exp \left(-0.8662 T_{e L(2)} a_{e R} m\right) \\
E_{e, d}= & x_{2} E_{\mathrm{ESNO}} \\
& \times\left[\begin{array}{c}
0.0065+\left(-0.045+0.0646 T_{e L(2)}\right) \cos \theta_{s} \\
-\left(-0.014+0.0327 T_{e L(2)}\right) \cos ^{2} \theta_{s}
\end{array}\right]
\end{aligned}
$$

where, $x_{1}$ and $x_{2}$ are the fraction of irradiance corresponding to the simulated spectral range, calculated through the spectral radiation model SMARTS2 [43], $E_{\mathrm{ESNO}}$ is the extraterrestrial normal irradiance, calculated as the product of the solar constant and the Sun-Earth correction factor, $\theta_{s}$ is the solar zenith angle obtained from the solar declination, pixel latitude and hour angle, $m$ is the relative optical air mass computed with the method of Kasten and Young [44], $a_{e r}$ is the optical thickness of a Rayleigh atmosphere parameterized by Louche et al. [45], and $T_{e L(2)}$ is the Linke turbidity factor. This last parameter is time and site specific and the model of Dumortier, [46], which describes the variations of turbidity over Western and Central Europe, is used to estimate it. 


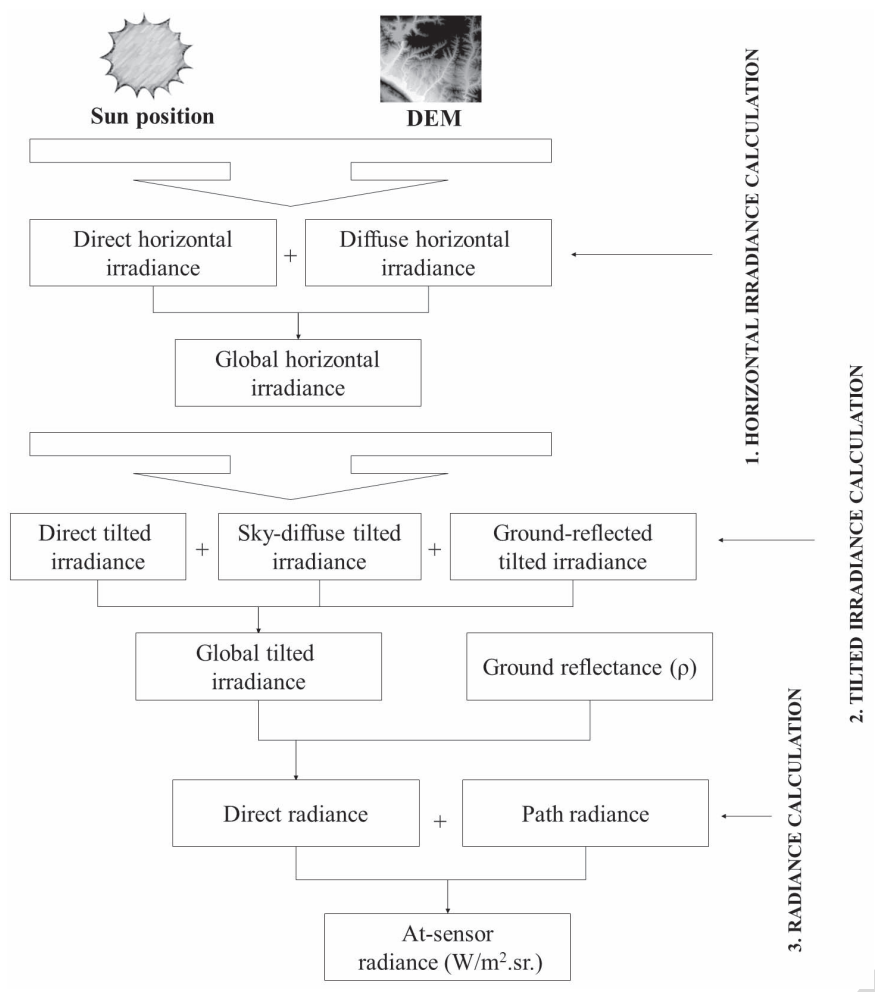

Fig. 1. Flowchart of synthetic scene generation procedure.

Obviously, the topography of Earth surface areas is normally nonflat, being necessary to consider the specific geometrical or topographical characteristics of each area. To compute the global tilted irradiance $\left(E_{\beta, g}\right)$, it is necessary to take into account not only the direct tilted irradiance or sunlight $\left(E_{\beta, s}\right)$, but also the ground-reflected irradiance $\left(E_{\beta, r}\right)$ as well as the sky diffuse irradiance or skylight $\left(E_{\beta, d}\right)$

$$
E_{\beta, g}=E_{\beta, s}+E_{\beta, d}+E_{\beta, r} .
$$

The first term, direct tilted irradiance $\left(E_{\beta, s}\right)$, is calculated applying the cosine law to direct horizontal irradiance. The effect of surrounding topography on direct radiation is modeled by adding a binary factor to control cast shadows proposed by Richter [47] $(0=$ shadow, $1=$ sunlit pixel $)$

$$
E_{\beta, s}=\Theta \frac{E_{e, s} \cos \gamma_{i}}{\cos \theta_{s}}
$$

where $E_{e, s}$ is the direct horizontal irradiance, calculated in (3), $\Theta$ is the cast shadow's binary factor, $\gamma_{i}$ is the solar incidence angle, and $\theta_{s}$ is the solar zenith angle.

The sky diffuse irradiance on an tilted plane is calculated with Hay's Model [48], also enhanced with the binary factor proposed by Richter. This term considers an isotropic and a circumsolar (anisotropic) component of diffuse irradiance

$$
E_{\beta, d}=E_{e, d}\left[\Theta \frac{A I \cos \gamma_{i}}{\cos \theta_{s}}+(1-\Theta A I) V_{d}\right]
$$

where, $E_{e, d}$ is the diffuse horizontal irradiance, calculated in (4), AI is Hay's anisotropy index, calculated from the ratio of direct irradiance on a surface normal to the sun's rays and the extraterrestrial normal irradiance, and $V_{d}$ is the sky view factor.
The sky view factor is based on Dozier's horizon algorithm [49], [50] and accounts for the portion of overlying hemisphere visible to a grid point depending on the terrain neighborhood of each pixel. The algorithm computes the vertical elevation angle of the horizon in $n$ directions to a specified radius. According to Dozier [50] $n=60$ is sufficient for radiation models. Similarly, for estimating the effect of topography on the solar irradiation received by the surface, the radius can generally be limited to $10 \mathrm{~km} \mathrm{[49].}$

The third term in (5), ground-reflected irradiance $\left(E_{\beta, r}\right)$, depends on the global irradiance impinging on the adjacent slopes, the reflectance of the surrounding objects, and the portion of adjacent terrain seen from a certain location

$$
E_{\beta, r}=E_{e, g \text { adj }} \rho_{\text {adj }} V_{t}
$$

where $E_{e, g \text { adj }}$ is the average global horizontal irradiance reaching the adjacent slopes in a square box of $0.5 \times 0.5 \mathrm{~km}, \rho_{\text {adj }}$ is the average terrain reflectance over a square box of the same size, and $V_{t}$ is the terrain view factor, that is, the portion of adjacent terrain seen from a certain location. $V_{d}$ and $V_{t}$ are complementary

$$
V_{d}(x, y)=1-V_{t}(x, y) .
$$

Finally, to generate the synthetic image, it is necessary to consider, in addition to $E_{\beta, g}$ and land-covers' reflectance, the orbital and observational configuration of the sensor, i.e., sensor viewing angle, spatial resolution of the sensor and acquisition time. The at-sensor radiance values can be calculated using the following expression:

$$
L=L_{p}+\frac{\rho T_{u} E_{\beta, g}}{\pi}
$$

where $L_{p}$ is the path radiance, i.e., radiation scattered into the sensor's instantaneous field of view without having ground contact, $\rho$ is the land-cover reflectance value, $T_{u}$ is the upward atmospheric transmittance, and $E_{\beta, g}$ is the global irradiance reaching each pixel. The path radiance is calculated by

$$
L_{p}=\frac{x_{3} E_{\mathrm{ESNO}} \cos \theta_{s} \rho^{\prime}{ }_{a}}{\pi} .
$$

where $x_{3}$ is a parameter representing the fraction of irradiance corresponding to the simulated spectral range, calculated through SMARTS2 spectral radiation model [43], $E_{\mathrm{ESNO}}$ is the solar extraterrestrial irradiance corrected by Sun-Earth distance, $\theta_{s}$ is the solar zenith angle, and $\rho^{\prime}{ }_{a}$ is the atmospheric albedo, calculated with Bird and Hulstrom's model [51] using values of aerosol's optical depth (AOD) for the considered area and date.

The direct upward atmospheric transmittance value $\left(T_{u}\right)$, depends, in turn, on the previously calculated optical thickness of the atmosphere, and the viewing angle of the satellite, and it is obtained through the following expression neglecting diffuse upward transmittance [52]:

$$
T_{u}=e^{0.8662 a_{e R} T_{c L(2)} / \cos \theta_{0}}
$$

where $a_{e R}$ is the optical thickness of the atmosphere and $\theta_{o}$ the viewing angle of the satellite. 


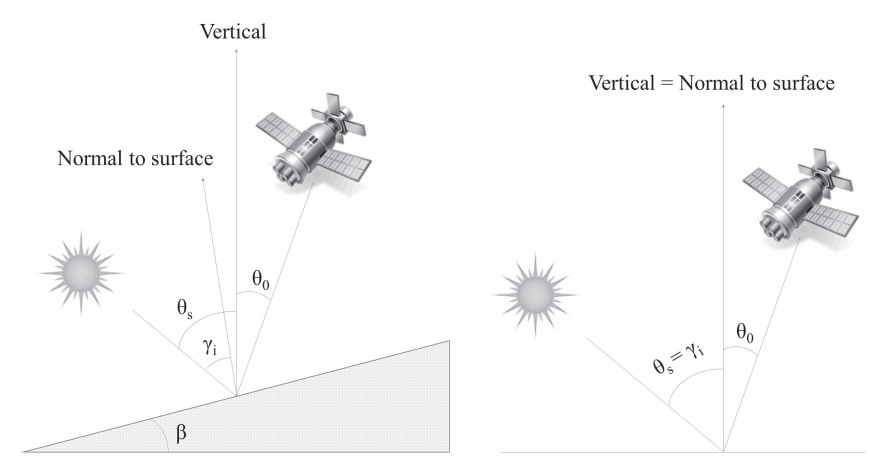

Fig. 2. Geometry on tilted and horizontal surfaces.

\section{B. Real (SR) and Horizontal (SH) Synthetic Images}

As already mentioned, a synthetic image represents the radiance an optical sensor would receive under specific geometric and temporal acquisition conditions, assuming a certain land-cover structure. Following the procedure proposed in Section III-A, it is possible to generate a synthetic image for a specific area considering its real relief or topography (SR image) or a synthetic image considering a completely flat topography (SH image). Geometry of both scenes is shown in Fig. 2. The comparison between the corrected image obtained applying a TOC method to an SR image and an SH image of the same area allows assessing the performance of the TOC applied.

The procedure to generate a $\mathrm{SH}$ image for a specific area is exactly the same to that applied to obtain an SR image, but considering horizontal DEM. When horizontal surfaces are simulated, the topographic effect is nonexistent, but there is still an influence of height on the atmospheric parameters involved in horizontal irradiance calculation. Consequently, horizontal irradiances are equal in both SR and SH calculation but for the former, topography affects the tilted irradiance calculation, unlike in $\mathrm{SH}$.

\section{Structural Similarity Index}

The synthetic image generated considering flat topography (SH) corresponds to the ideal TOC correction, when the topographic distortions disappear entirely. To measure the similarity between this mentioned ideal correction $\mathrm{SH}$ and the TOC-corrected SR images, the structural similarity index (SSIM) is used. The SSIM is a quantitative metric that gives relatively accurate similarity prediction [53], which correlates well with perceptual image fidelity [54]. This index is an improved version of the universal quality index [55]; proposed by Wang et al. [56], and has gained widespread popularity because of its simple formulation and its applicability to different image processing tasks, e.g., image compression [57], pan-sharpening [58]-[60], image denoising [61], [62], image restoration [63], [64], or downscaling [60]. The SSIM index, considers three different components of similarity: Luminance comparison, contrast comparison, and structural similarity. Therefore, it provides a more complete similarity measure than individual statistics such as RMSE or the correlation coefficient $\left(r_{x, y}\right)$

$$
\operatorname{SSIM}_{(x, y)}=\left(l_{(x, y)}^{\alpha}\right)\left(c_{(x, y)}^{\beta}\right)\left(s_{(x, y)^{\gamma}}\right)
$$

where $\operatorname{SSIM}_{(x, y)}$ is the structural similarity index between two images $x$ and $y ; l_{(x, y)}$ is the luminance component, calculated as a function of the means $\mu_{x}$ and $\mu_{y} ; c_{(x, y)}$ is the contrast component, depending on the standard deviations $\sigma_{x}$ and $\sigma_{y}$; $s_{(x, y)}$ is the structure component, based on the correlation coefficient $r_{x y} ; \alpha>0, \beta>0, \gamma>0$ are parameters used to adjust the relative importance of the three components. $1_{(x, y) \text {, }}$ $c_{(x, y)}$, and $s_{(x, y)}$ are calculated using the equations proposed in [56]. Coefficients $\alpha, \beta$, and $\gamma$ are set to 1 to simplify the expression, as the authors proposed. In this case, (13) reduces to

$$
\operatorname{SSIM}_{(x, y)}=\frac{\left(2 \mu_{x} \mu_{y}+C_{1}\right)\left(2 \sigma_{x y}+C_{2}\right)}{\left(\mu_{x}^{2}+\mu_{y}^{2}+C_{1}\right)\left(\sigma_{x}^{2}+\sigma_{y}^{2}+C_{2}\right)}
$$

where $\mu_{i}$ is the mean value of the image $i, \sigma_{i}$ is its standard deviation, and $\sigma_{i j}$ is the covariance of $i$ and $j . C_{1}$ and $C_{2}$ are two user-defined constants included to avoid unstable results when $\mu_{x}{ }^{2}+\mu_{y}{ }^{2}$ and $\sigma_{x}{ }^{2}+\sigma_{y}{ }^{2}$ are very close to zero. In our case, $\mathrm{C} 1$ and $\mathrm{C} 2$ are set to 0.065 and 0.585 , respectively, following recommendations by Wang et al. [55]. These values are somewhat arbitrary, but the performance of the SSIM index is demonstrated fairly insensitive to variations of these values [55].

The SSIM index is normally used for comparing an ideal reference image (in our case $\mathrm{SH}$ ), with a distorted or erroneous one (in our case TOC-corrected SR). Its dynamic range is $[-1,1]$. The best value 1 is obtained only when perfect similarity is achieved.

In practice, one usually requires a single overall quality measure of the entire image [56]. We use a mean SSIM (MSSIM) index to evaluate the overall image quality. MSSIM can be used to quantitatively rank the performance of TOC methods. In addition, for image quality assessment, it is useful to apply the SSIM index locally rather than globally [65], computing the local statistics within an $11 \times 11$ circularsymmetric Gaussian weighting function which moves pixelby-pixel over the image [54]. A combination of MSSIM index and SSIM maps will provide a useful tool to select the best TOC depending on the subsequent use of the corrected images.

\section{CASE STUdY}

\section{A. Study Area and Field Data}

As already pointed out, synthetic images can be generated considering different topographic, geometric, and temporal configurations, as well as different land-cover distributions. Rough topography can be responsible for topography-related image distortions, while terrain slope and aspect can influence the natural spectral variability within any land-cover type [4]. Therefore, this case study is carried out on a mountainous area (Pyrenees) of the northeastern side of Navarre, Spain, where the relief is rough and the valleys have a wide variety of aspects (see Fig. 3).

The study area considered has an extension of $155 \mathrm{~km}^{2}$, with heights ranging between 430 and $1110 \mathrm{~m}$, and slopes from $0^{\circ}$ to $81^{\circ}$. For this area, a 5-m resolution DEM of the region of Navarre obtained through standard photogrammetric techniques is available. From this DEM, terrain aspect and 


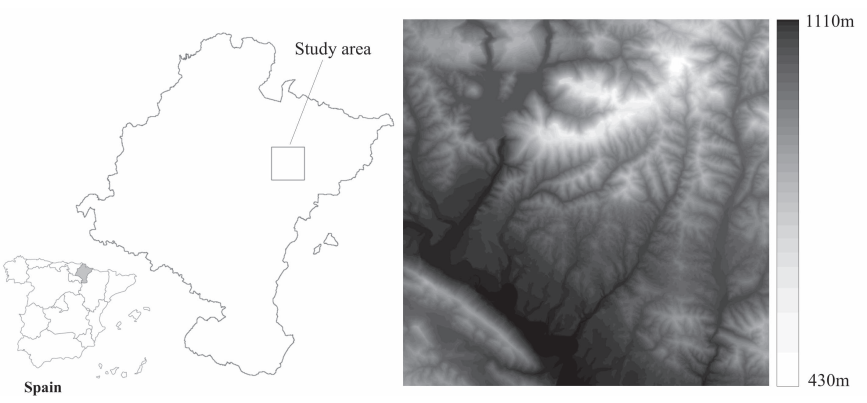

Fig. 3. Study area of $13 \times 13 \mathrm{~km}$ in northern Navarre and DEM available.

slope are calculated over a $3 \times 3$ cell neighborhood, through an averaging process from altitude differences within the grid in both " $x$ " and " $y$ " directions. The date, time, and acquisition configuration parameters selected for this case study are those of a hypothetical scene acquired the 15th of February of 2009, at 10:45 UTC. A winter time acquisition is selected for this case study to show strong alterations due to the topographic effect.

Concerning the sensor configuration, we considered a panchromatic sensor with a spectral range between 500 and $900 \mathrm{~nm}$, typical of panchromatic wide range sensors, with a spatial resolution of $5 \mathrm{~m}$ and a nadiral viewing angle. To obtain a land-cover reflectance image as realistic as possible, land-use cartography is used as well as spectral information from 20 different land-covers obtained from spectral libraries of ASTER and USGS for vegetation, rocks and soil [66], [67]. The study area is mainly covered by conifer forests (25\%), deciduous and mixed forests (24\%), herbaceous crops (21\%), shrubs (14\%), and grasslands and pasture lands (3\%).

The parameterization of reflectance used here requires reference land-cover information, which might not be available in the general case. This type of parameterization was selected in our case in order to adequately validate the technique proposed. Other simpler parameterizations could be followed, e.g., considering constant reflectance throughout the scene, leading to more unrealistic, yet simple, synthetic images. Preliminary analyses suggest that the influence of reflectance parameterization is only minor in the TOC evaluation procedure proposed here.

In the next subsection, the horizontal and tilted irradiance images and the SR and SH images generated are described. The computational time for the simulation of synthetic real and horizontal images in an area of $155 \mathrm{~km}^{2}$ is about $2 \mathrm{~h}$ 10 min, using a Intel Core 2 Quad CPU Q8400 $2.66 \mathrm{GHz}$, 3.49-Gb RAM, being the computation of the sky view factor the most time-consuming task in the process.

\section{B. Synthetic Images Obtained}

The main parameters involved in the calculation of the horizontal irradiance, in (2)-(4), both for SR and SH images, for our particular case study are shown in Table II.

The global horizontal irradiance $\left(E_{e, g}\right)$ is the sum of both direct and diffuse components, and its values range from 235.0 to $248.5 \mathrm{~W} / \mathrm{m}^{2}$, either for both SR and SH, being the variations
TABLE II

VAlues of PARAMETERS ReQuired For the GeNeration of GLOBAL HORIZONTAL IRRADIANCE IMAGES (SR AND SH)

\begin{tabular}{c|cccc}
\hline \hline & \multicolumn{2}{|c}{ Synthetic Real (SR) } & \multicolumn{2}{c}{ Synthetic Horizontal (SH) } \\
\cline { 2 - 5 } Parameters & $\mu \pm \sigma$ & Range & $\mu \pm \sigma$ & Range \\
$h(\mathrm{~m})$. & $646 \pm 133$ & {$[435-1110]$} & $646 \pm 133$ & {$[435-1110]$} \\
$\gamma_{s}\left({ }^{\circ}\right)$ & $30.6 \pm 0.1$ & {$[30.4-30.8]$} & $30.6 \pm 0.1$ & {$[30.4-30.8]$} \\
Variables & & & & \\
$E_{e, s}\left(\mathrm{~W} / \mathrm{m}^{2}\right)$ & $201 \pm 3.9$ & {$[194.7-214.6]$} & $201 \pm 3.9$ & {$[194.7-214.6]$} \\
$E_{e, d}\left(\mathrm{~W} / \mathrm{m}^{2}\right)$ & $39 \pm 1.3$ & {$[34.0-40.3]$} & $39 \pm 1.3$ & {$[34.0-40.3]$} \\
\hline $\boldsymbol{E}_{e, g}\left(\mathbf{W} / \mathbf{m}^{2}\right)$ & $239 \pm 2.7$ & {$[235.0-248.5]$} & $239 \pm 2.7$ & {$[235.0-248.5]$} \\
\hline \hline
\end{tabular}

TABLE III

VALUES OF PARAMETERS REQUiRED FOR THE GENERATION of GLOBAL TILTED IRRADIANCE

\begin{tabular}{c|cccc}
\hline \hline & \multicolumn{2}{|c}{ Synthetic Real (SR) } & \multicolumn{2}{c}{ Synthetic Horizontal (SH) } \\
\hline Parameters & $\mu \pm \sigma$ & Range & $\mu \pm \sigma$ & Range \\
\cline { 2 - 5 }$\beta\left(^{\circ}\right)$ & $16.2 \pm 10$ & {$[0-76]$} & 0 & {[]} \\
$\rho$ & $0.42 \pm 0.13$ & {$[0.2-0.63]$} & $0.42 \pm 0.13$ & {$[0.2-0.63]$} \\
$V_{d}$ & $0.83 \pm 0.08$ & {$[0.12-1]$} & 1 & {[]} \\
$\cos \gamma_{i}$ & $0.49 \pm 0.2$ & {$[-0.71-1]$} & 0.509 & {[]} \\
Variables & & & & \\
$E_{\beta, s}\left(\mathrm{~W} / \mathrm{m}^{2}\right)$ & $193.4 \pm 78.7$ & {$[0-415.2]$} & $200.6 \pm 3.9$ & {$[194.7-214.6]$} \\
$E_{\beta, r}\left(\mathrm{~W} / \mathrm{m}^{2}\right)$ & $15.6 \pm 6.8$ & {$[0-79.3]$} & 0 & {[]} \\
$E_{\beta, d}\left(\mathrm{~W} / \mathrm{m}^{2}\right)$ & $33.4 \pm 3.8$ & {$[3.4-42.2]$} & $200.6 \pm 3.9$ & {$[34.0-40.3]$} \\
$\boldsymbol{E}_{\beta, g}\left(\mathbf{W} / \mathbf{m}^{2}\right)$ & $242 \pm 80.2$ & {$[28.4-482.1]$} & $239 \pm 2.7$ & {$[235.0-248.5]$} \\
\hline \hline
\end{tabular}

of irradiance mainly caused by the effect of altitude on the different atmospheric parameters involved.

Next, the three components of global tilted irradiance $\left(E_{\beta, g}\right)$, shown in Fig. 5, are computed using direct, diffuse, and global horizontal irradiances. In flat terrain $(\mathrm{SH})$, the ground-reflected irradiance, $E_{\beta, r}$, is zero. As a result, $E_{\beta, g}$ is obviously the same as the global horizontal irradiance, due to the flat terrain.

For the SR image, summing the three terms mentioned, in (5), a global tilted irradiance image with values ranging from 28.5 to $482.1 \mathrm{~W} / \mathrm{m}^{2}$ is obtained, with a mean value of $241.8 \mathrm{~W} / \mathrm{m}^{2}$ and a standard deviation of 80.2 (see Table III).

When flat terrain is considered slope is obviously zero, and therefore there is no ground-reflected irradiance.

In Fig. 4, some of the factors included in the synthetic image calculation are shown, such as the sky view factor $\left(V_{d}\right)$, the binary factor controlling cast shadows $(\Theta)$, the cosine of solar incidence angle, and the image of reflectances used.

Obviously except for the reflectance image, the others have a constant value for the SH image, as they are terrain dependent. $V_{d}$ is 1 across the whole image meaning a clear sky hemisphere for every pixel. In the absence of sloped surfaces, there is no need to control any shadow, therefore $\Theta$ is 1 as well. In addition, in flat terrain the solar incidence angle is equal to the solar zenith angle for every pixel.

Finally, synthetic images are obtained using (8), considering the ground reflectance image and the previously mentioned sensor configuration parameters (Fig. 5). The SR image shows values of radiance between 9.8 and $90.5 \mathrm{~W} / \mathrm{m}^{2}$.sr., with a mean of $37.1 \mathrm{~W} / \mathrm{m}^{2}$.sr and a standard deviation of 


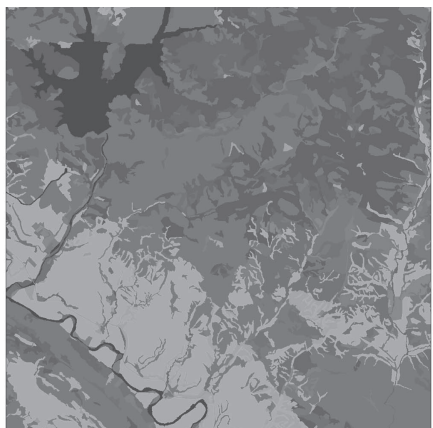

(a)

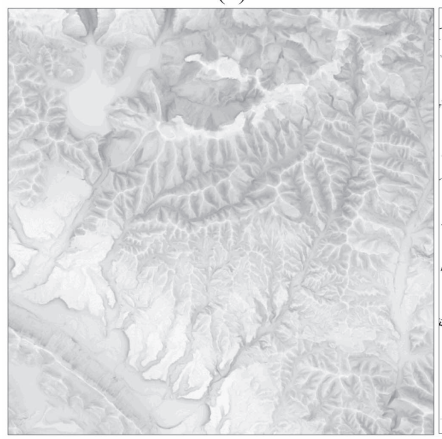

(c)

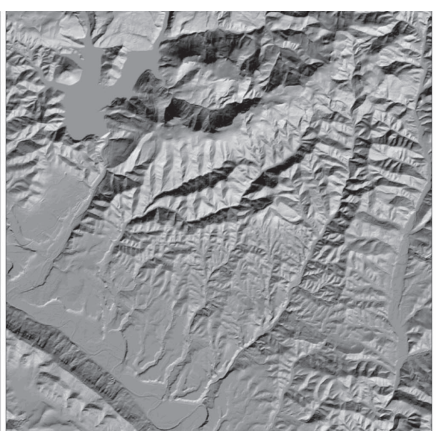

(b)

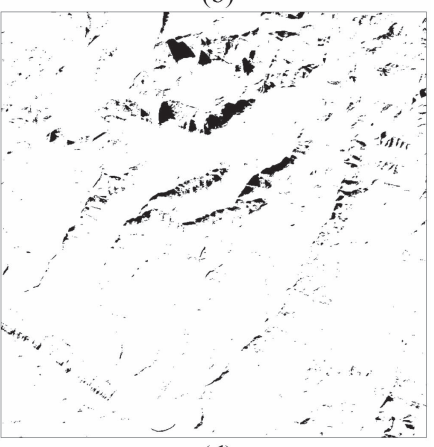

(d)

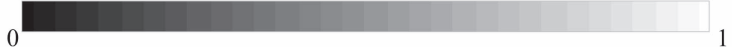

Fig. 4. Images of parameters used in the calculation of synthetic image when real relief is considered (SR). (a) Land-cover's reflectance. (b) Cosine of solar incidence angle $\left(\cos \gamma_{i}\right)$. (c) Image sky view factor $\left(V_{d}\right)$. (d) Binary factor $(\Theta)$ controlling cast shadows.

TABLE IV

VALUES OF Synthetic IMAGES. SyNTHETIC REAL (SR) AND S YNTHETIC HORIZONTAL ( $\mathrm{SH}$ )

\begin{tabular}{c|cccc}
\hline \hline \multirow{2}{*}{ Variables } & \multicolumn{2}{|c}{ Synthetic Real (SR) } & \multicolumn{2}{c}{ Synthetic Horizontal (SH) } \\
\cline { 2 - 5 } & $\mu \pm \sigma$ & Range & $\mu \pm \sigma$ & Range \\
\cline { 2 - 5 } $\mathrm{L}_{\mathrm{e}}\left(\mathrm{W} / \mathrm{m}^{2} . \mathrm{sr}\right)$. & $29.3 \pm 13.1$ & {$[2.0-82.8]$} & $28.6 \pm 8.6$ & {$[13.5-43.8]$} \\
$\mathrm{L}_{\mathrm{p}}\left(\mathrm{W} / \mathrm{m}^{2} . \mathrm{sr}\right)$ & $7.77 \pm 0.02$ & {$[7.72-7.81]$} & $7.77 \pm 0.02$ & {$[7.72-7.81]$} \\
\hline $\begin{array}{c}\text { Synthetic image } \\
\left(W / \mathrm{m}^{2} \text {.sr.) }\right.\end{array}$ & $37.1 \pm 13.1$ & {$[9.8-90.5]$} & $36.4 \pm 8.6$ & {$[21.3-51.5]$} \\
\hline \hline
\end{tabular}

13.06 (Table IV). On the other hand, the $\mathrm{SH}$ image ranges from 21.3 to $51.5 \mathrm{~W} / \mathrm{m}^{2}$.sr., being the mean $36.4 \mathrm{~W} / \mathrm{m}^{2}$.sr. and a standard deviation of 8.6. The differences between SR and $\mathrm{SH}$ are only due to the topographic effect, which leads to variations in different radiance components (Table IV).

As seen in Table IV, the direct radiance is the main component of the resultant synthetic scenes, and the influence of topography on it is obvious, since variance is clearly higher in the SR scene. This topographic effect should be corrected by TOC algorithms.

\section{RESUlts AND Discussion}

The algorithms tested in this paper are the empiricalstatistical method [4], the $C$-correction [4], the enhanced Minnaert including slope [3], and the cosine method [4]. Their formulation is shown in Table $\mathrm{V}$, where A and B are, respectively, the intercept and the slope of the regression line between radiance and illumination, i.e., $\cos \gamma_{i}$, and $k$ and $C$

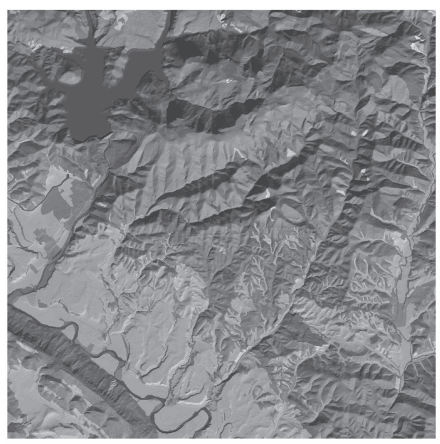

(a)

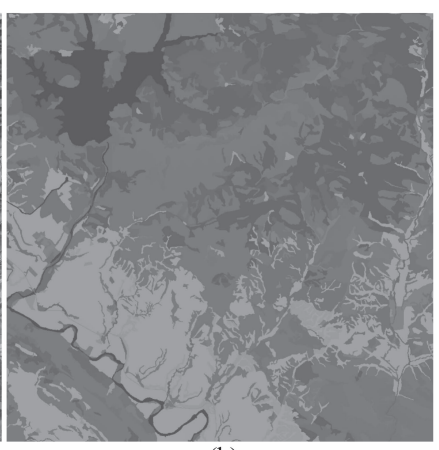

(b)

$90 \mathrm{~W} / \mathrm{m}^{2}$. sr.
$0 \mathrm{~W} / \mathrm{m}^{2} . \mathrm{sr}$

Fig. 5. (a) Synthetic image (SR), when real topography is considered. (b) Synthetic horizontal ( $\mathrm{SH})$ image, when flat topography is considered.

TABLE V

Formulation of TOC Method TESTED

\begin{tabular}{c|c}
\hline \hline TOC method & Formula \\
\hline \hline Cosine & $L_{\text {corr }}=L \frac{\cos \theta_{s}}{\cos \gamma_{i}}$ \\
C-Correction & $L_{\text {corr }}=L \frac{\cos \theta_{s}+C}{\cos \gamma_{i}+C}$ \\
Enhanced Minnaert & $L_{\text {corr }}=L\left[\frac{\cos ^{k} \theta_{s}}{\cos ^{k} \gamma_{i} \cos ^{k-1} \beta}\right]$ \\
Empirical-Statistical & $L_{\text {corr }}=L-\left(A+B \cos \gamma_{i}\right)+L_{\text {mean }}$ \\
\hline \hline
\end{tabular}

are empiric constants calculated for each method as described by Teillet et al. [4].

These four TOC methods are selected for being probably some of the most frequently used in the literature. In addition, their differences will hopefully provide contrasting results for discussing the utility of the proposed evaluating method.

In Fig. 6, the SR-corrected images using the four TOC methods selected are shown, including a zoom area to see in detail some of the most problematic areas in the image.

Areas where the solar incidence angle is close or even higher than $90^{\circ}$ are normally not corrected because most TOC methods are unstable at these low $\cos \gamma_{i}$ values [25]. However, the $\gamma_{i}$ boundary might be different for each TOC due to differences in their approach and formulation. In this paper, when the cosine method is applied, pixels with $\gamma_{i}>85^{\circ}$ are left uncorrected. This $85^{\circ}$ angle boundary was proposed as a limit for excluding shadowed areas by Baraldi et al. [25]. When $\gamma_{i} \in\left[90^{\circ}, 180^{\circ}\right]$, corrected radiance is negative, which has no physical meaning [25], and when $\gamma_{i} \in\left[85^{\circ}, 90^{\circ}\right]$, the pixel information is low, and TOC with the cosine method produces strong overcorrection [21]. In our particular case, those pixels represent the $5 \%$ of the image. The enhanced Minnaert method uses logarithms on its equation to compute $k$ constant. Thus, areas with $\cos \gamma_{i} \leq 0$ cannot be corrected with this method, leading to $1.6 \%$ of the pixels masked out in this case. In the case of the $C$-correction method, this boundary can be relaxed because its formulation already introduces a $C$ factor to reduce overcorrection. So, in order to avoid negative radiance values in the computations, a boundary depending on 

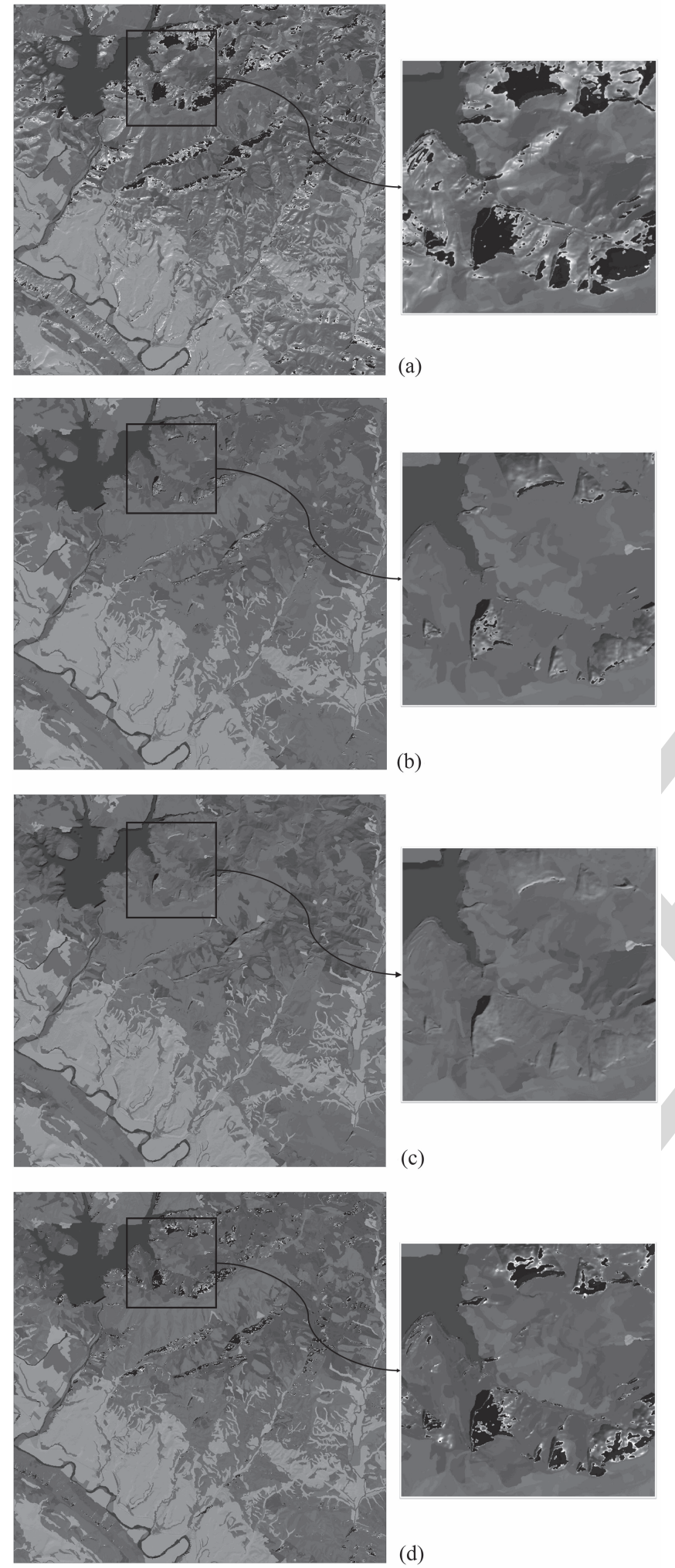

(d)

$0 \mathrm{~W} / \mathrm{m}^{2} . \mathrm{sr}$.

$90 \mathrm{~W} / \mathrm{m}^{2} . \mathrm{sr}$

Fig. 6. TOC-corrected images using the four different TOC algorithms selected. (a) Cosine method. (b) $C$-correction method. (c) Empirical-statistical method. (d) Enhanced Minnaert method.

the $C$ factor obtained for each particular case is proposed. In our case, pixels with $\cos \gamma_{i} \leq-C / 2$ are masked out for the $C$-correction method, those pixels represent the $0.2 \%$ of the pixels. Finally, the empirical-statistical method does not have any limitations with this regard and no areas of the image need to be masked out before the correction, so it can be applied to $100 \%$ of the image.

Those uncorrected pixels (areas in black in Fig. 6), form areas with low radiance values, equal to those of the original SR image, and contrast with the surrounding pixels, which might still be over-corrected with some methods. In particular, overcorrection is still noticed when the cosine method is used, leading to bright zones surrounding dark uncorrected areas [Fig. 6(a)]. Some overcorrection is also present in the enhanced Minnaert corrected image [Fig. 6(d)], although much less than in the cosine method. Finally, the $C$-correction method and particularly the empirical-statistical method gives better results in these problematic areas with almost negligible overcorrection effects. It must be remarked that because of the particular extreme conditions of our simulated images, regarding acquisition date and time, these problems of overcorrection are particularly severe.

At a first sight, it is quite easy to appreciate differences between the TOC-corrected images obtained with each method (Fig. 6). On the one hand, visually, the $C$-correction and empirical-statistical methods appear as the most successful in reducing the topographic effect in the original SR image [Fig. 6(b) and (c)]. The former's performance seems slightly better, although the latter has the advantage of correcting every pixel in the image, with no signal of overcorrection. On the other hand, the cosine method does not achieve a proper correction of the shadowed areas, as mentioned above. Finally, the enhanced Minnaert method seems to successfully correct the effect of topography in general [Fig. 6(d)], but overcorrection is observed in some pixels.

Apart from the visual assessment, a quantitative evaluation is performed using the SSIM index [56] to accurately determine the quality of the corrected images. An SSIM index map of the area is generated for each TOC method (Fig. 7).

The SSIM maps generated for each TOC-corrected image show the performance of the correction pixel by pixel. It is easy to appreciate the poorer correction of the cosine method, while $C$-correction method performs better than other TOC-s, but still has problems to successfully correct pixels where $\cos \gamma_{i}$ is close to zero or even negative. So, although the $C$-correction method corrects most of the pixels in the image, leading to visually appealing results, the corrected radiances obtained for areas of low $\cos \gamma_{i}$ are still quite different from what they should, and give low SSIM values. These areas with low SSIM values are also obtained in the empirical-statistical correction and, to a much larger extent, in the enhanced Minnaert correction. Flat areas (e.g., lower left of the image) and south facing slopes are normally adequately corrected with most methods. Areas with moderate slopes are corrected better with the empirical-statistical method, and especially with the $C$-correction method.

In practice, one usually requires a single overall quality measure of the entire image [56]. We use a mean SSIM (MSSIM) index to evaluate the overall image quality.

The MSSIM indexes obtained comparing the SH image and the TOC-corrected SR images, are shown in Table VI. 


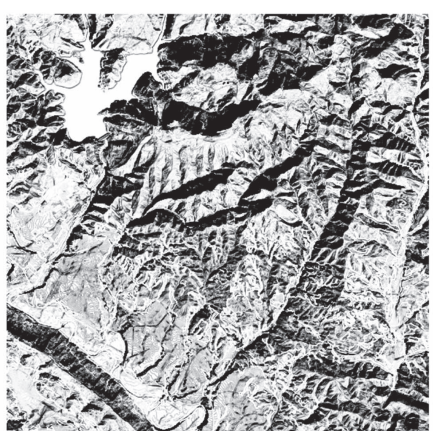

(a)

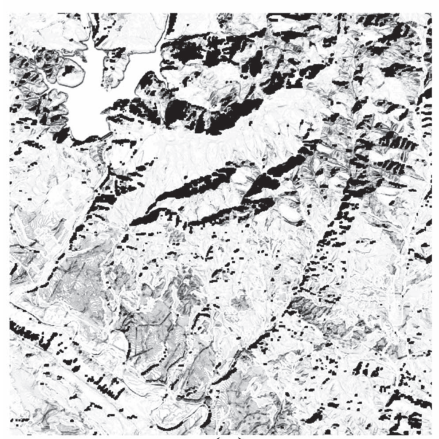

(c)

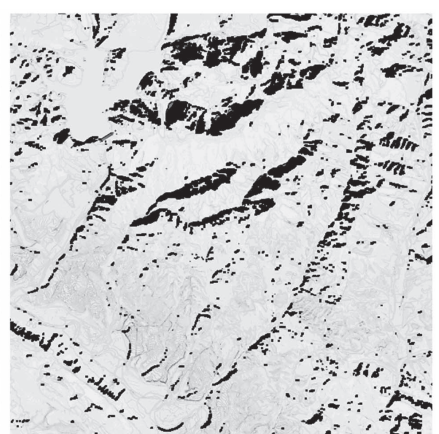

(b)

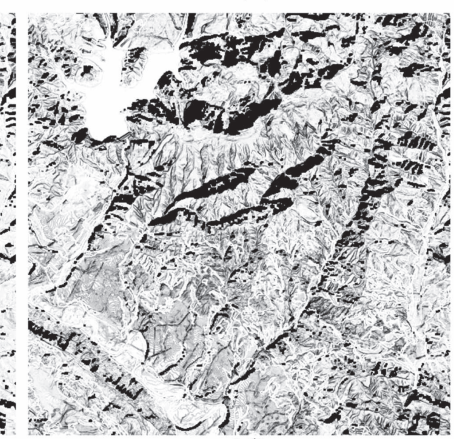

(d)

0 1

Fig. 7. SSIM index maps computed using a moving window of $11 \times 11$ pixels for the four method selected. (a) Cosine method. (b) $C$-correction method. (c) Empirical-statistical method. (d) Enhanced Minnaert method.

TABLE VI

Mean SSIM Values AND OTHER Similarity Measures ObTAINEd For THE Four TOC Methods TEsted

\begin{tabular}{cc|cccc}
\hline \hline & & \multicolumn{4}{|c}{ TOC METHODS } \\
& $\boldsymbol{S R}$ & $\boldsymbol{C O S}$ & $\boldsymbol{C}$-COR & $\boldsymbol{E M P I}$ & $\boldsymbol{M I N}$ \\
MSSIM & 0.466 & 0.584 & 0.889 & 0.820 & 0.783 \\
$\mathbf{I}_{(\mathbf{x}, \mathbf{y})}$ & 0.966 & 0.979 & 0.998 & 0.997 & 0.995 \\
$\mathbf{c}_{(\mathbf{x}, \mathbf{y})}$ & 0.544 & 0.637 & 0.906 & 0.850 & 0.811 \\
$\mathbf{S}_{(\mathbf{x}, \mathbf{y})}$ & 0.884 & 0.878 & 0.969 & 0.959 & 0.938 \\
$\mathbf{R M S E}$ & 8.556 & 9.795 & 2.824 & 2.622 & 8.549 \\
$\boldsymbol{\Delta} \hat{\boldsymbol{\sigma}}$ & -0.205 & -0.185 & -0.034 & -0.030 & -0.179 \\
$\mathbf{r}_{(\mathbf{x}, \mathbf{y})}$ & 0.765 & 0.654 & 0.962 & 0.961 & 0.728 \\
\hline \hline
\end{tabular}

For comparison, along with the MSSIM its three components; luminance $l_{(x, y)}$, contrast $c_{(x, y)}$, and structure $s_{(x, y)}$, are shown in Table VI, as well as other statistical indexes to compare each image pair, such as the coefficient of correlation $\left(r_{x y}\right)$, the root mean square error (RMSE), and normalized standard deviation difference $(\Delta \widehat{\sigma})$. The latter represents the normalized difference in standard deviation, i.e., $\left(\sigma_{x}-\sigma_{y}\right) /\left(\sigma_{x}+\sigma_{y}\right)$, with 0 representing two images with the same standard deviation.

According to MSSIM the original SR image (no TOC correction) shows a similarity of 0.466 with the ideal $\mathrm{SH}$ image. This value is improved with all the four TOC methods tested. On the one hand, cosine method ranks last, only slightly improving the original image. On the other hand, $C$-correction methods perform best with a MSSIM value higher than 0.88 in the corrected scene. The enhanced Minnaert and the empiricalstatistical methods give intermediate MSSIM values.

The quantitative evaluation and ranking of TOC methods can be analyzed in more detail looking at the values of

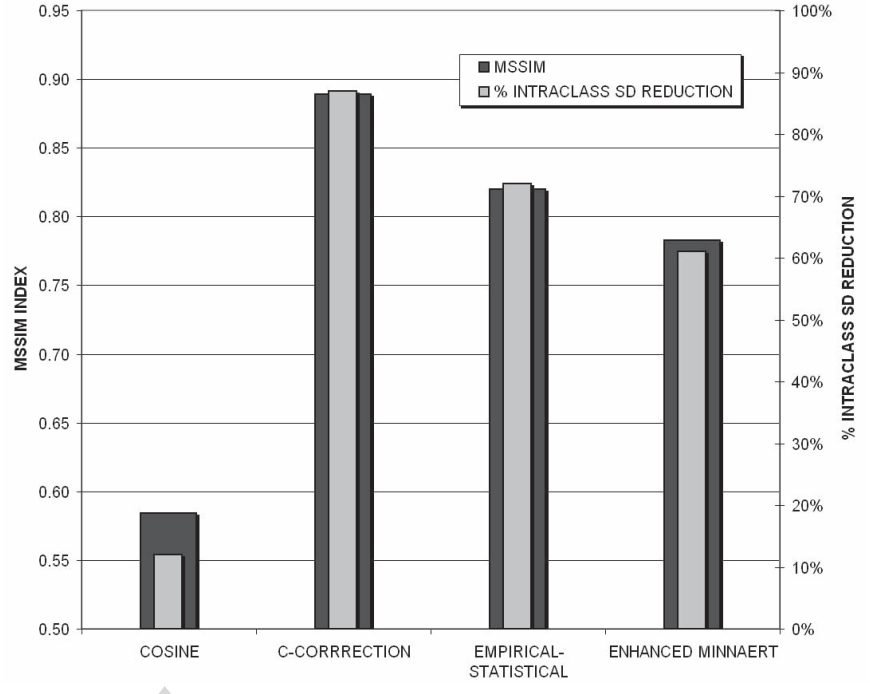

Fig. 8. MSSIM index compared with percentage of reduction of intraclass standard deviation.

the three SSIM components. For example, $C$-correction and empirical-statistical are the best methods according to the luminance, contrast and structure comparison, result confirmed by the values of RMSE, $r_{x, y}$ and $\Delta \widehat{\sigma}$, where these methods ranked first and second for all of them. On the contrary, the cosine method ranks last for all the six criteria considered.

SR is used to refer to SR noncorrected image. COS refers to cosine method, $C$-COR is $C$-correction method, EMPI is empirical-statistical method, and MIN is enhanced Minnaert method.

The three components of SSIM are conceptually related to the RMSE, $\Delta \widehat{\sigma}$ and $r_{x, y}$, respectively, but they do not represent exactly the same magnitudes (i.e., their equations are related but not the same). Therefore, the rankings of TOC methods obtained with $l_{(x, y)}, c_{(x, y)}$, and $s_{(x, y)}$ on the one hand and RMSE, $\Delta \widehat{\sigma}$ and $r_{x, y}$ on the other might not be exactly the same.

Finally, to compare the TOC evaluation procedure proposed here with other assessment approaches used traditionally, the reduction of the standard deviation of land-cover classes has been computed and compared with the MSSIM results (Fig. 8).

As already explained in Section II-B, a traditional indirect procedure to assess the goodness of TOC corrections is via the reduction of the standard deviation within each land-cover class. Successful TOC algorithms will result in more homogeneous land-covers, allowing a better accuracy in subsequent classifications. In Fig. 8, the average reduction of the standard deviation of classes (in percent) is shown for the four TOC methods tested, along with their MSSIM value. The correspondence between both criteria is clear, with the $C$-Correction method ranking first, followed by the empiricalstatistical, the enhanced Minnaert and the cosine method. These results confirm the validity of the MSSIM-based TOC evaluation procedure proposed here.

Finally, comparing the results of the TOC evaluation performed here with those of the literature, we can find an overall agreement. The simplistic hypothesis of cosine method, considering only direct irradiance, was also found inappropriate 
in previous studies [8], [13], [30]. The cosine method has frequently shown a problem of over-correction, particularly when the algorithm is applied for correcting steep, naturally vegetated slopes [9], this is only partially avoided by introducing a limit incident angle, above which no correction is done.

Similarly to [6] and [9], we observed that only small differences exists among $C$-correction, empirical-statistical, and enhanced Minnaert methods. In contrast to our results, Twele and Erasmi [9] observed the best correction of enhanced Minnaert in natural tropical forests, slightly better than the other nonLambertian approaches, i.e., empirical-statistical and $C$-correction. In that study [9], TOC performance is measured using as a criteria the reduction in coefficient of variation and linear regression analysis between corrected data and $\cos \gamma_{i}$.

Alternatively, Riaño et al. [12] observed that most TOC methods produced an overcorrection where $\cos \gamma_{i}$ is low, even if they worked with a summer scene, which had good illumination conditions. Enhanced Minnaert method did not give acceptable results in their studies, modifying the mean of the original scene. In addition, $C$-correction showed better results than enhanced Minnaert method according to the reduction of intraclass variation, which is consistent with our paper (Fig. 8).

When the image is taken under unfavorable illumination conditions, Hantson and Chuvieco [10] observed the empirical-statistical method gave the best results for bare soil pixels, and to a lesser extent the $C$-correction. These two methods gave the best results for pine forest pixels as well. The performance of TOC methods was evaluated via the reduction of standard deviation of pixel values within the same landcover in different slopes and aspects [17].

In general, other studies concluded that the empiricalstatistical and the $C$-correction methods gave the most adequate results, retaining the spectral characteristics of the data, homogenizing land-covers and improving overall classification accuracy [10], [12], [68], [69]. In this paper, the $C$-correction method resulted in the best TOC correction for the study area and the acquisition conditions considered. This result is confirmed both by the MSSIM index and the reduction of intraclass deviation. It must be remarked that the scene acquisition date and time considered here are representative of winter scenes, where sun illumination is lowest. Analogous to [10], we observed that when the image is taken at a low sun elevation angle, no TOC method is able to correct entirely the topographic effect.

\section{CONCLUSION}

This paper presented an objective and universal procedure to evaluate the quality of TOC methods applied to remote sensing imagery. The approach proposed is based on the use of synthetic images, which can be generated for a certain area and acquisition conditions considering both real topography (SR image) or a completely flat topography (SH image). The latter is not affected by illumination differences caused by topography and, therefore, can be considered a reference against which to compare TOC-corrected SR scenes. The comparison of TOC-corrected SR scenes and the reference SH was carried out using a widely accepted quality metrics, i.e., the SSIM index [56]. This index quantitatively evaluated several aspects of image similarity and can be used to build a ranking of best performing TOC methods for each specific case.

The approach presented several advantages compared with traditional evaluation techniques. First, the approach is objective because the results of each TOC method were quantitatively evaluated and ranked using the SSIM index. Second, the approach did not necessarily require ancillary information on land-cover distribution to perform the TOC quality assessment. Finally, synthetic images representing different settings and scene acquisition conditions can be generated to select the best performing TOC for each particular situation (e.g., solar angles, spatial resolution, etc.). Accordingly, the influence of each configuration parameter on the performance of the TOC methods can be evaluated. Obviously, this approach assumes that a TOC showing a good performance for synthetic imagery also performs correctly for real imagery with similar acquisition configuration.

The case study analyzed here, considering a winter panchromatic scene, showed similar results using the approach proposed and the assessment of the reduction of the intraclass standard deviation (a traditional TOC evaluation procedure). In particular, $C$-correction method ranked first, followed by the empirical-statistical and the enhanced Minnaert methods. The cosine method achieved the poorest TOC correction. The SSIM can be applied locally to detect the areas where TOC methods perform the worst. In this case, areas with low-illumination conditions showed the worst results with all methods. The combination of SSIM maps and mean SSIM index provided a useful tool to decide the best TOC according to the future use of the corrected scene.

Further research is needed to apply the proposed technique over a range of sensor (e.g., spatial resolution, band frequency, etc.) and acquisition (e.g., acquisition date and time) configurations, to derive guidelines on which the TOC method performs best under each situation. Therefore, the technique proposed, can be used to perform a detailed analysis of the accuracy of existing TOC methods.

\section{REFERENCES}

[1] S. A. Soenen, D. R. Peddle, and C. A. Coburn, "SCS+C: A modified sun-canopy-sensor topographic correction in forested terrain," IEEE Trans. Geosci. Remote Sens., vol. 43, no. 9, pp. 2148-2159, Sep. 2005.

[2] D. Lu, H. Ge, S. He, A. Xu, G. Zhou, and H. Du, "Pixel-based Minnaert correction method for reducing topographic effects on a Landsat 7 ETM+ image," Photogram. Eng. Remote Sens., vol. 74, no. 11, pp. 1343-1350, Nov. 2008.

[3] J. A. Smith, T. L. Lin, and K. J. Ranson, "The Lambertian assumption and landsat data," Photogram. Eng. Remote Sens., vol. 46, no. 9, pp. 1183-1189, Sep. 1980

[4] P. M. Teillet, B. Guindon, and D. G. Goodenough, "On the slope-aspect correction of multispectral scanner data," Can. J. Remote Sens., vol. 8, no. 12, pp. 84-106, Dec. 1982.

[5] D. L. Civco, "Topographic normalization of Landsat Thematic Mapper digital imagery," Photogram. Eng. Remote Sens., vol. 55, no. 9, pp. 1303-1309, Sep. 1989

[6] P. Meyer, K. I. Itten, T. Kellenberger, S. Sandmeier, and R. Sandmeier, "Radiometric corrections of topographically induced effects on Landsat TM Data in an alpine environment," ISPRS J. Photogram. Remote Sens., vol. 48, no. 4, pp. 17-28, Aug. 1993. 
[7] D. S. Lu, H. L. Ge, S. Z. He, A. J. Xu, G. M. Zhou, and H. Q. Du, "Pixel-based Minnaert correction method for reducing topographic effects on a Landsat 7 ETM+ image," Photogram. Eng. Remote Sens., vol. 74, Nov. pp. 1343-1350, Nov. 2008.

[8] K. H. Law and J. Nichol, "Topographic correction for differential illumination effects on IKONOS satellite imagery," Int. Soc. Photogram. Remote Sens., vol. 35, pp. 641-646, Jan. 2004.

[9] A. Twele and S. Erasmi, "Evaluating topographic correction algorithms for improved land cover discrimination in mountainous areas of central Sulawesi," Remote Sens. GIS Environ. Stud., Appl. Geograph. Göttingen Geograph. Essays, vol. 113, pp. 287-295, Jan. 2005.

[10] S. Hantson and E. Chuvieco, "Evaluation of different topographic correction methods for Landsat imagery," Int. J. Appl. Earth Observat. Geoinf., vol. 13 no. 5, pp. 691-700, May 2011.

[11] N. Ghasemi, A. Mohammadzadeh, and M. R. Sahebi, "Assessment of different topographic correction methods in ALOS AVNIR-2 data over a forest area," Int. J. Digital Earth, pp. 1-17, Oct. 2011, DOI: $10.1080 / 17538947.2011 .625049$

[12] D. Riano, E. Chuvieco, J. Salas, and I. Aguado, "Assessment of different topographic corrections in Landsat-TM data for mapping vegetation types," IEEE Trans. Geosci. Remote Sens., vol. 41, no. 5, pp. 1056-1061, May 2003

[13] A. Twele, M. Kappas, J. Lauer, and S. Erasmi, "The effect of stratified topographic correction on land cover classification in tropical mountainous regions," in Proc. 7th Symp. ISPRS Commun., 2006, pp. 432-437.

[14] M. Minnaert, "The reciprocity principle in lunar photometry," Astrophys. J., vol. 93, pp. 403-410, May 1941.

[15] D. Gu and A. Gillespie, "Topographic normalization of Landsat TM images of forest based on subpixel sun-canopy-sensor geometry," Remote Sens. Environ., vol. 64, no. 2, pp. 166-175, May 1998.

[16] Y. N. Gao and W. C. Zhang, "Variable empirical coefficient algorithm for removal of topographic effects on remotely sensed data from rugged terrain," in Proc. IEEE Int. Geosci. Remote Sens. Symp., Jul. 2007, pp. 4733-4736.

[17] S. Kobayashi and K. Sanga-Ngoie, "A comparative study of radiometric correction methods for optical remote sensing imagery: The IRC vs. other image-based C-correction methods," Int. J. Remote Sens., vol. 30, no. 2, pp. 285-314, 2009

[18] M. Vincini, D. Reeder, and E. Frazzi, "An empirical topographic normalization method for forest TM data," in Proc. IEEE Int. Geosci. Remote Sens. Symp., Jun. 2002, pp. 2091-2093.

[19] R. Richter, T. Kellenberger, and H. Kaufmann, "Comparison of topographic correction methods," Remote Sens., vol. 1, no. 3, pp. 184-196, Jul. 2009.

[20] R. Richter, "Correction of satellite imagery over mountainous terrain," Appl. Opt., vol. 37, no. 18, pp. 4004-4015, Jun. 1998.

[21] T. Tokola, J. Sarkeala, and M. Van der Linden, "Use of topographic correction in Landsat TM-based forest interpretation in Nepal," Int J. Remote Sens., vol. 22, no. 4, pp. 551-563, Mar. 2001.

[22] M. P. Bishop and J. D. Colby, "Anisotropic reflectance correction of SPOT-3 HRV imagery," Int. J. Remote Sens., vol. 23, no. 10, pp. 2125-2131, 2002.

[23] M. P. Bishop, J. F. Shroder, Jr., and J. D. Colby, "Remote sensing and geomorphometry for studying relief production in high mountains," Geomorphology, vol. 55, nos. 1-4, pp. 345-361, Sep. 2003.

[24] S. Ekstrand, "Landsat TM-based forest damage assessment: Correction for topographic effects," Photogram. Eng. Remote Sens., vol. 62 pp. 151-161, Feb. 1996

[25] A. Baraldi, M. Gironda, and D. Simonetti, "Operational two-stage stratified topographic correction of spaceborne multispectral imagery employing an automatic spectral-rule-based decision-tree preliminary classifier," IEEE Trans. Geosci. Remote Sens., vol. 48, no. 1, pp. 112-146, Jan. 2010.

[26] H. Reese and H. Olsson, "C-correction of optical satellite data over alpine vegetation areas: A comparison of sampling strategies for determining the empirical C-parameter," Remote Sens. Environ., vol. 115, no. 6 , pp. 1387-1400, Jun. 2011.

[27] M. Törmä and P. Härmä, "Topographic correction of Landsat ETM images in Finnish Lapland," in Proc. IEEE Int. Geosci. Remote Sens. Symp., Jul. 2003, pp. 3629-3631.

[28] K. I. Itten and P. Meyer, "Geometric and radiometric correction of TM-Data of mountainous forested areas," IEEE Trans. Geosci. Remote Sens., vol. 31, no. 4, pp. 764-770, Jul. 1993.

[29] C. Conese, M. A. Gilabert, F. Maselli, and L. Bottai, "Topographic normalization of TM scenes through the use of an atmospheric correction method and digital terrain models," Photogram. Eng. Remote Sens., vol. 59, no. 12, pp. 1745-1753, Dec. 1993.
[30] W. C. Zhang and Y. N. Gao, "LULC classification and topographic correction of Landsat-7 ETM+ imagery in the Yangia River Watershed: The influence of DEM resolution," Sensors, vol. 9, no. 3, pp. 1980-1995, Mar. 2009.

[31] J. D. Shepherd and J. R. Dymond, "Correcting satellite imagery for the variance of reflectance and illumination with topography," Int. J. Remote Sens., vol. 24, no. 17, pp. 3503-3514, Sep. 2003.

[32] M. Parente, J. Trevor Clark, A. J. Brown, and J. L. Bishop, "End-to-end simulation and analytical model of remote-sensing systems: Application to CRISM," IEEE Trans. Geosci. Remote Sens., vol. 48 no. 11, pp. 3877-3888, Nov. 2010.

[33] A. Borner, L. Wiest, P. Keller, R. Reulke, R. Richter, M. Schaepman, and D. Schläpferb, "SENSOR: A tool for the simulation of hyperspectral remote sensing systems," ISPRS J. Photogram. Remote Sens., vol. 55, nos. 5-6, pp. 299-312, Mar. 2001.

[34] (2006). The DIRSIG User's Manual [Online]. Available: http://dirsig.org/docs/manual/index.html

[35] L. Guanter, K. Segl, and H. Kaufmann, "Simulation of optical remotesensing scenes with application to the enmap hyperspectral mission," IEEE Trans. Geosci. Remote Sens., vol. 47, no. 7, pp. 2340-2351, Jul. 2009.

[36] H. C. Hottel and A. Whiller, "Evaluation of fiat plate solar collector performance," in Proc. Present. Conf. Solar Energy, Jul. 1958, pp. 1-6.

[37] B. Y. H. Liu and R. C. Jordan, "The interrelationship and characteristic distribution of direct, diffuse and total solar radiation," Solar Energy, vol. 4, no. 3, pp. 1-19, Jul. 1960.

[38] C. C. Y. Ma and M. Iqbal, "Statistical comparison of solar radiation correlation Monthly average global and diffuse radiation on horizontal surfaces," Solar Energy, vol. 33, no. 2, pp. 143-148, 1984.

[39] Handbook of Fundamentals, American Society of Heating, Refrigeration and Air-Conditioning Engineers, Inc., Atlanta, GA, USA, 1985.

[40] J. Page, "Algorithms for the SATELLIGHT programme," The European Database of Daylight and Solar Radiation, Bergen, Norway, Tech. Rep. 2, Jun. 1996.

[41] P. Ineichen, "Radiation derivation from meteosat counts," The European Database of Daylight and Solar Radiation, Freiburg, Germany, Tech. Rep. 6, Sep. 1998.

[42] D. Dumortier, Mesure, Analyse et Modélisation du Gisement Lumineux. Application à L'évaluation Des Performances de L'éclairage Naturel Des Bâtiments. Chambry, France: Univ. Chambry, 1995.

[43] C. Gueymard, SMARTS2, a Simple Model of the Atmospheric Radiative Transfer of Sunshine: Algorithms and Performance Assessment. Cocoa, FL, USA: Florida Solar Energy Center, Dec. 1995.

[44] F. Kasten and A. T. Young, "Revised optical air mass tables and approximation formula," Appl. Opt., vol. 28, no. 22, pp. 4735-4738, Nov. 1989.

[45] A. Louche, G. Peri, and M. Iqbal, "An analysis of the Linke turbidity factor," Solar Energy, vol. 37, no. 6, pp. 393-396, 1986.

[46] D. Dumortier, "The SATELLIGHT model of turbidity variations in Europe," Ecole Nationale des Travaux Publics de l'Etat, Freiburg, Germany, Tech. Rep. 6, Sep. 1998

[47] R. Richter, "Correction of satellite imagery over mountainous terrain," Appl. Opt., vol. 37, no. 18, pp. 4004-4015, Jun. 1998.

[48] J. E. Hay and D. C. McKay, "Estimating solar irradiance on inclined surfaces: A review and assessment of methodologies," Int. J. Solar Energy, vol. 3, nos. 4-5, pp. 230-240, 1985.

[49] K. Zakšek, K. Oštir, and Ž. Kokalj, "Sky-view factor as a relief visualization technique," Remote Sens., vol. 3, no. 2, pp. 398-415, 2011.

[50] J. Dozier, J. Bruno, and P. Downey, "A faster solution to the horizon problem," Comput. Geosci., vol. 7, no. 2, pp. 145-151, 1981.

[51] R. E. Bird and R. L. Hulstrom. (1981). A simplified clear sky model for direct and diffuse insolation on horizontal surfaces [Online]. Available: http://www.nrel.gov/rredc/pdfs/761.pdf

[52] M. A. Gilabert, C. Conese, and F. Maselli, "An atmospheric correction method for the automatic retrieval of surface reflectances from TM images," Int. J. Remote Sens., vol. 15, no. 10, pp. 2065-2086, Jul. 1994.

[53] S. Rezazadeh and S. Coulombe, "A novel approach for computing and pooling Structural SIMilarity index in the discrete wavelet domain," in Proc. 16th IEEE Int. Conf. Image Process., Nov. 2009, pp. 2209-2212.

[54] D. Brunet, E. R. Vrscay, and Z. Wang, "On the mathematical properties of the structural similarity index," IEEE Trans. Image Process., vol. 21, no. 4, pp. 1488-1499, Apr. 2012.

[55] Z. Wang and A. C. Bovik, "A universal image quality index," IEEE Signal Process. Lett., vol. 9, no. 3, pp. 81-84, Mar. 2002.

[56] Z. Wang, A. C. Bovik, H. R. Sheikh, and E. P. Simoncelli, "Image quality assessment: From error visibility to structural similarity," IEEE Trans. Image Process., vol. 13, no. 4, pp. 600-612, Apr. 2004. 
[57] L. Bo, Y. Rui, and J. Hongxu, "Remote-sensing image compression using two-dimensional oriented wavelet transform," IEEE Trans. Geosci. Remote Sens., vol. 49, no. 1, pp. 236-250, Jan. 2011.

[58] Y. Ling, M. Ehlers, E. L. Usery, and M. Madden, "FFT-enhanced IHS transform method for fusing high-resolution satellite images," ISPRS J. Photogram. Remote Sens., vol. 61, no. 6, pp. 381-392, Feb. 2007.

[59] M. Ehlers, S. Klonus, P. Johan Åstrand, and P. Rosso, "Multi-sensor image fusion for pansharpening in remote sensing," Int. J. Image Data Fusion, vol. 1, no. 1, pp. 25-45, Mar. 2010.

[60] V. F. Rodriguez-Galiano, E. Pardo-Igúzquiza, M. Chica-Olmo, J. Mateos, J. P. Rigol-Sánchez, and M. Vega, "A comparative assessment of different methods for Landsat 7/ETM+ pansharpening," Int. J. Remote Sens., vol. 33, no. 20, pp. 6574-6599, Oct. 2012.

[61] C. Yue and W. Jiang, "SAR image denoising in nonsubsampled contourlet transform domain based on maximum a posteriori and non-local constraint," Remote Sens. Lett., vol. 4, no. 3, pp. 270-278, Mar. 2012.

[62] Q. Yuan, L. Zhang, and H. Shen, "Hyperspectral image denoising employing a spectral-spatial adaptive total variation model," IEEE Trans. Geosci. Remote Sens., vol. 50, no. 10, pp. 3660-3677, Oct. 2012.

[63] O. Jeromin and M. S. Pattichis, "Multiscale sampling geometries and methods for deterministic and stochastic reconstructions of magnitude and phase spectra of satellite imagery," IEEE Trans. Geosci. Remote Sens., vol. 50, no. 10, pp. 3678-3692, Oct. 2012.

[64] M. Soccorsi, D. Gleich, and M. Datcu, "Huber-Markov model for complex SAR image restoration," IEEE Geosci. Remote Sens. Lett., vol. 7, no. 1, pp. 63-67, Jan. 2010.

[65] Z. Wang, A. C. Bovik, and E. P. Simoncelli, Handbook of Image and Video Processing, 2nd ed. Linn, MO, USA: Elsevier Inc., 2005, pp. 1355-1372.

[66] A. M. Baldridge, S. J. Hook, C. I. Grove, and G. Rivera, "The ASTER spectral library version 2.0," Remote Sens. Environ., vol. 113, no. 4, pp. 711-715, Apr. 2009.

[67] R. N. Clark, G. A. Swayze, R. Wise, E. Livo, T. Hoefen, R. Kokaly, and S. J. Sutley. (2007). USGS Digital Spectral Library Splib06a: U.S. Geological Survey, Digital Data Series 231 [Online]. Available: http://speclab.cr.usgs.gov/spectral.lib06

[68] E. R. McDonald, X. Wu, and P. A. Caccetta, "Illumination correction of Landsat TM data in south east NSW," in Proc. 10th Australasian Remote Sens. Conf., Aug. 2000, pp. 1-13.

[69] W. C. Zhang and Y. N. Gao, "A simple empirical topographic correction method for ETM+ imagery," Int. J. Remote Sens., vol. 30, no. 9, pp. 2259-2275, Jun. 2009.

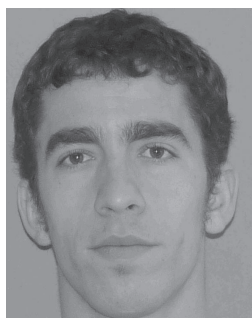

Ion Sola received the Surveying Technical Engineering degree from the University of the Basque Country, Gasteiz, Spain, in 2006, and the Masters degree in environmental engineering from the University of Santiago de Compostela, Santiago de Compostela, Spain, in 2008. His Ph.D. thesis focused on topographic correction of remote sensing imagery.

He is currently a Research Fellow with the Department of Projects and Rural Engineering, Public University of Navarre, Iruñea, Spain.

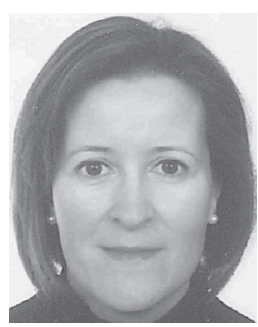

María González-Audícana received the M.S. and $\mathrm{Ph} . \mathrm{D}$. degrees in agricultural engineering from the Public University of Navarre, Pamplona, Spain, in 1996 and 2001, respectively. Her thesis is based on image fusion.

She has been a Professor with the Department of Projects and Rural Engineering, Public University of Navarre since 1997. Her current research interests include the area of remote-sensing processing. She is currently involved in research activities on topographic effect correction, multisensor data fusion, and image spectral and textural classification for agricultural management purposes.

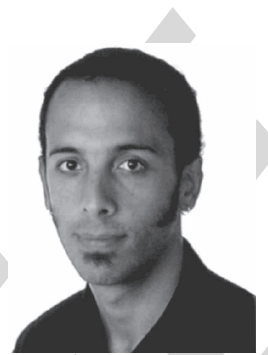

Jesus Álvarez-Mozos received the Engineering and $\mathrm{Ph} . \mathrm{D}$. degrees in agricultural engineering from the Public University of Navarre, Iruñea, Spain, in 2001 and 2006, respectively.

He was a Teaching Assistant from 2001 to 2010. Since 2010, he has been an Associate Professor with the Department of Projects and Rural Engineering, Public University of Navarre. His current research interests include remote sensing data processing and hydrological applications of remote sensing.
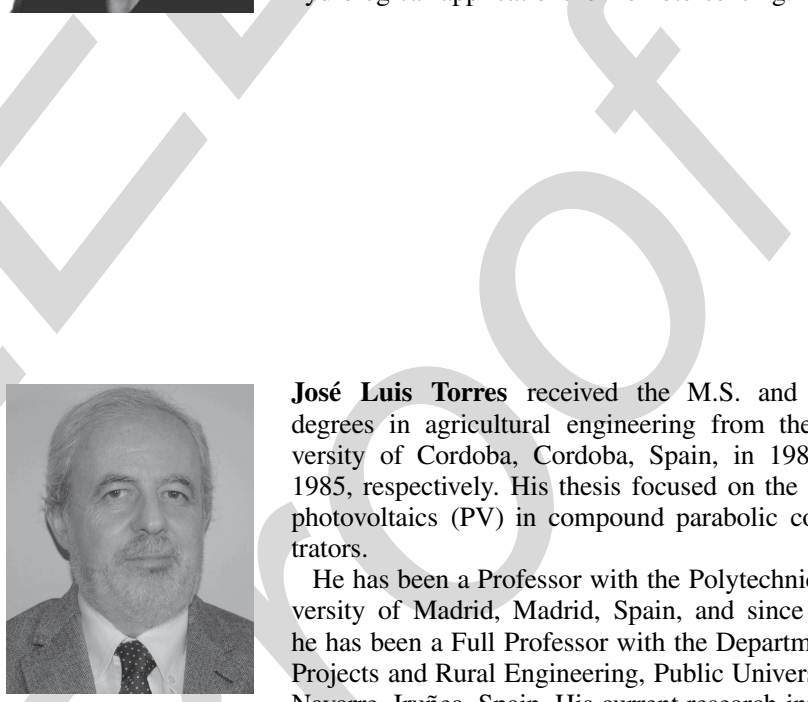

José Luis Torres received the M.S. and Ph.D. degrees in agricultural engineering from the University of Cordoba, Cordoba, Spain, in 1981 and 1985, respectively. His thesis focused on the use of photovoltaics (PV) in compound parabolic concentrators.

He has been a Professor with the Polytechnic University of Madrid, Madrid, Spain, and since 1991, he has been a Full Professor with the Department of Projects and Rural Engineering, Public University of Navarre, Iruñea, Spain. His current research interests include evaluation of renewable energy resources and PV. 
Please be aware that the authors are required to pay overlength page charges $(\$ 200$ per page) if the paper is longer than 6 pages. If you cannot pay any or all of these charges please let us know.

This pdf contains 2 proofs. The first half is the version that will appear on Xplore. The second half is the version that will appear in print. If you have any figures to print in color, they will be in color in both proofs.

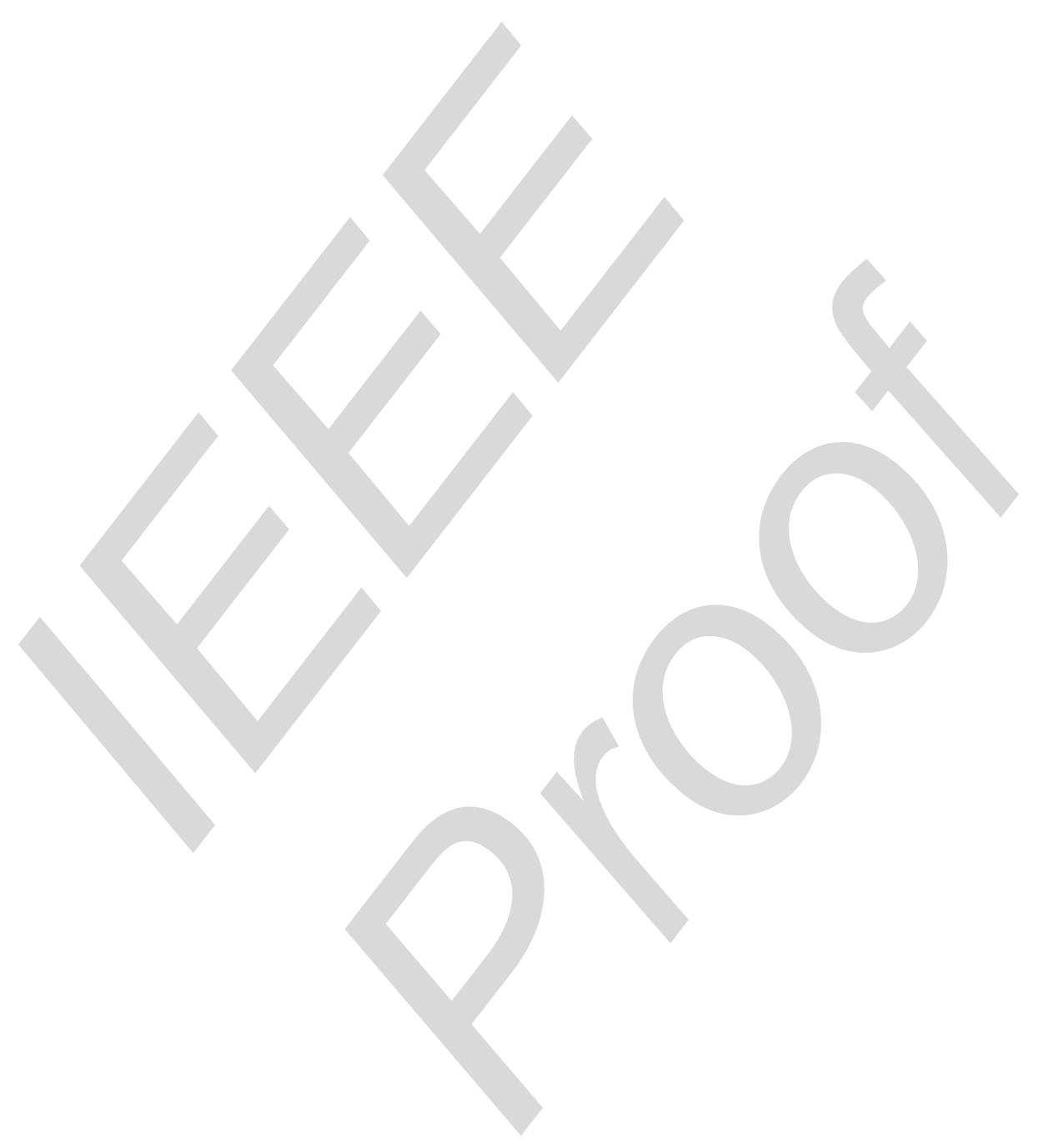

